\title{
Metallomics
}

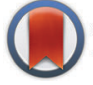

CrossMark <click for updates

Cite this: Metallomics, 2017 9, 411

Received 17th January 2017 Accepted 7th February 2017

DOI: $10.1039 / c 7 m t 00012 j$

rsc.li/metallomics

\section{Whole-brain metallomic analysis of the common marmoset (Callithrix jacchus)}

\author{
B. Knauer, ${ }^{\text {ab }}$ P. Majka, ${ }^{\text {C K. J. Watkins, }}{ }^{\text {a A. W. R. Taylor, }}{ }^{a}$ D. Malamanova, ${ }^{a}$ B. Paul, ${ }^{\text {de }}$ \\ Hsin-Hao Yu, ${ }^{a}$ A. I. Bush, ${ }^{e}$ D. J. Hare $\dagger^{\text {ef }}$ and D. H. Resert‡*ag
}

\begin{abstract}
Despite the importance of transition metals for normal brain function, relatively little is known about the distribution of these elemental species across the different tissue compartments of the primate brain. In this study, we employed laser ablation-inductively coupled plasma-mass spectrometry on PFA-fixed brain sections obtained from two adult common marmosets. Concurrent cytoarchitectonic, myeloarchitectonic, and chemoarchitectonic measurements allowed for identification of the major neocortical, archaecortical, and subcortical divisions of the brain, and precise localisation of iron, manganese, and zinc concentrations within each division. Major findings across tissue compartments included: (1) differentiation of white matter tracts from grey matter based on manganese and zinc distribution; (2) high iron concentrations in the basal ganglia, cortex, and substantia nigra; (3) co-localization of high concentrations of iron and manganese in the primary sensory areas of the cerebral cortex; and (4) high manganese in the hippocampus. The marmoset has become a model species of choice for connectomic, aging, and transgenic studies in primates, and the application of metallomics to these disciplines has the potential to yield high translational and basic science value.
\end{abstract}

\section{Introduction}

Transition metals are widely recognized as essential components of normal brain function, and disruption of metal homeostasis is implicated in a wide array of clinically important neuropathologies. ${ }^{1-5}$ However, the anatomical distribution of metals in the brain is relatively poorly understood, and to date, wholebrain metal maps have only been generated for invertebrates ${ }^{6}$ and mice, ${ }^{7}$ among widely used laboratory species. This presents difficulties for interpretation of metallomic changes in human disease states, given the well-known differences in rodent and

\footnotetext{
${ }^{a}$ Department of Physiology, Faculty of Medicine, Nursing, and Health Sciences, Monash University, Clayton, Victoria, 3800, Australia

${ }^{b}$ Research School, Ruhr University Bochum, Bochum, Germany

${ }^{c}$ Laboratory of Neuroinformatics, Department of Neurophysiology,

Nencki Institute of Experimental Biology of Polish Academy of Sciences,

3 Pasteur Street, 02-093 Warsaw, Poland

${ }^{d}$ School of Earth Sciences, The University of Melbourne, Parkville, Victoria, 3010, Australia

${ }^{e}$ The Florey Institute of Neuroscience and Mental Health, The University of Melbourne, Parkville, 3010, Victoria, Australia

${ }^{f}$ Elemental Bio-imaging Facility, University of Technology Sydney, Broadway, NSW, Australia

${ }^{g}$ Graduate Entry Medical Program, School of Rural Health, Monash University, Churchill, Victoria, 3842, Australia

$\dagger$ These authors made equal intellectual contributions to this work.

\$ Bldg. 13F, Physiology, Monash University, Clayton, VIC 3800, Australia. E-mail: david.reser@monash.edu
}

primate neuroanatomy. Using laser ablation-inductively coupled plasma-mass spectrometry (LA-ICP-MS), we examined the brain of the common marmoset (Callithrix jacchus), an increasingly important model of human neurological disease. ${ }^{8-13}$

Recent mapping studies in mice ${ }^{7}$ have shown that there is heterogeneous distribution of manganese, iron, copper and zinc across the nuclei and anatomical compartments of the mammalian brain, but information regarding metals in other species, at different ages, and for other metals, ranges from sparse to nonexistent. The detailed mouse metallome generated by Paul et al., showed clearly that spatial distribution of metals in the brain is related to the functional properties of regions with enriched or impoverished concentrations. In that study, there was a clear relationship between iron enrichment and high concentrations of tyrosine hydroxylase (the rate limiting synthetic enzyme for catecholamine neurotransmitters) in areas such as the substantia nigra and olfactory bulbs. Furthermore, through image alignment and thresholding for each metal tested, they were able to generate a dynamic, 3-dimensional map which highlighted different tissue compartments, depending on the target. Thus, by filtering for high or low signal strength of particular metals, specific regions (e.g. hippocampus, cortex, or basal ganglia) could be demarcated based on their metallomic characteristics.

Generation of a dynamic, 3-dimensional, user-controlled anatomical atlas represents a genuine advance in functional anatomy, and it is clear from the work performed in the mouse 
that this goal is attainable in the near future. However, the utility of such a tool is limited if it is confined to a single species, even one as ubiquitous as the laboratory mouse. The difficulties of single-gene and single-species approaches to research into neurological conditions is well documented and have been recently reviewed, ${ }^{14}$ and there are several critical differences in structural anatomy between the rodent and human brain. These include, but are not limited to: unification of the caudate and putamen regions of the striatum; ${ }^{15,16}$ a disproportionate increase in the volume of white matter; ${ }^{17}$ reduced or absent homologous areas of the prefrontal $\operatorname{cortex}^{18}$ and posterior cingulate; ${ }^{19}$ and reduced neuron density as a function of body mass. ${ }^{20}$ There are also functional differences which make it difficult to interpret rodent findings in terms of primate brain processes, including increased corticalisation of sensory functions and the appearance of 'new' cortical areas in primates. ${ }^{21}$

Ideally, a metallomic map of the mammalian brain would include one or more non-human primate species, for both comparative anatomical and translational research purposes. However, the size and complexity of the primate brain place severe constraints upon the spatial resolution of mapping techniques, including LA-ICP-MS, to the point that a reasonably complete map of the entire brain of a macaque or human is not currently feasible. The marmoset provides an attractive alternative, in that the brain volume is only moderately larger than that of a rat, yet it has all of the structural and functional characteristics of the primate order. These features facilitate LA-ICP-MS scanning, and our laboratory has extensive experience with structural and functional studies in marmosets. ${ }^{22-25}$ In this report, we present the first whole-brain studies of transition metal distribution in a non-human primate, with special attention to changes in relative metal concentration across the known anatomical boundaries and tissue compartments of the forebrain. Our findings indicate the marmoset represents a desirable and practical model species for generation of a meso-scale metallome of the primate brain.

\section{Methods}

\section{Sample preparation}

Brains were obtained from five adult marmosets used for in vivo electrophysiological experiments in our laboratory. All in vivo procedures were approved in advance by the Monash University Animal Ethics Committee, and conformed to the Australian National Health and Medical Research Council Guide for Care and Use of Laboratory Animals, which strongly encourages use of archived and scavenged tissues where possible. Case data for the animals included in this study are presented in Table 1. At the conclusion of the electrophysiological recordings, all animals were humanely euthanised (sodium pentobarbital, $100 \mathrm{mg} \mathrm{kg}^{-1}$, i.m.) and transcardially perfused with $0.1 \mathrm{M}$ PBS followed by $4 \%$ PFA (except M1598, see below). The brains were extracted and post-fixed overnight in 4\% PFA, then cryoprotected in serially increasing concentrations of sucrose (10-30\%). Cryoprotected brains were blocked and sectioned ( $40 \mu \mathrm{m}$ thickness) on a cryostat. Adjacent sections were allotted for myelin, ${ }^{26}$ Nissl (cresyl violet),
Table 1 Case information for marmosets included in this report

\begin{tabular}{llll}
\hline Case_ID & Age (y) & Sex & BW (g) \\
\hline CJ154 & 3 & F & 457 \\
CJ166 & 2 & F & 369 \\
F1510 & 2 & F & 420 \\
M1477 & M & 386 \\
M1598 & 4 & M & 327 \\
\hline
\end{tabular}

cytochrome oxidase, ${ }^{27}$ and calbindin staining, or for LA-ICP-MS. Sections intended for LA-ICP-MS scanning were mounted uncoverslipped on double-sized microscope slides, protected from light and dust, dried, and stored for 1-40 weeks prior to scanning.

Histological sections were digitally scanned at $20 \times$ magnification using an aperio AT-2 slide scanner, and the resulting high-resolution images were extracted, segmented, and re-sized, then imported into Adobe Illustrator (v. CS-6, Adobe Systems, Mountain View, CA) for alignment with the LA-ICP-MS images.

Cortical flat mount. Case M1598 was initially perfused with $2 \%$ PFA, and the brain was immediately removed and processed using a modification of the approach developed by Tootell, et $a l^{28}$ The right hemisphere was blocked behind the caudal terminus of the lateral sulcus, and the underlying white matter and subcortical nuclei were gently removed by blunt dissection. Two small cuts were made in the remaining cortical sheet, and the sheet was then gently pressed flat under a glass microscope slide. The cortical sheet and slide were weighted with a glass beaker in a petri dish filled with $2 \%$ PFA for 24 hours. The flat mounted cortex was then cryoprotected as described above, sectioned at $40 \mu \mathrm{m}$ thickness. Adjacent sections were processed for cytochrome oxidase or mounted unstained and uncoverslipped for LA-ICP-MS scanning.

\section{Selection and identification of anatomical targets}

Transition metal concentrations were evaluated across eleven tissue compartments in the marmoset brain. In this instance, 'compartment' refers to an anatomical area identifiable in both histological sections and in the carbon-13 scan of the LA-ICP-MS output. Broadly defined compartments included: cerebral cortex; white matter tracts (including subcortical white matter, corpus callosum, internal and external capsules, and corona radiata); amygdaloid complex; basal ganglia (which in practice comprised the striatum and globus pallidus); claustrum; hippocampus (all subregions); selected thalamic nuclei (pulvinar and lateral geniculate); septum, superior colliculus, and substantia nigra. The pulvinar and lateral geniculate nuclei of the thalamus were selected as preliminary indicators of thalamic metal composition, as it was not possible to evaluate each individual thalamic nucleus at the current scanning resolution, due to the heavy intercalation of white matter tracts between thalamic subnuclei. Fig. 1 illustrates the ROI boundaries and corresponding anatomical compartments in a representative carbon-13 parasagittal section from case CJ-166, along with the corresponding Nissl and myelin stained sections.

\section{Parcellation of cerebral cortex}

The marmoset brain includes a relatively large cortical surface area, and primary sensory areas which are topographically 

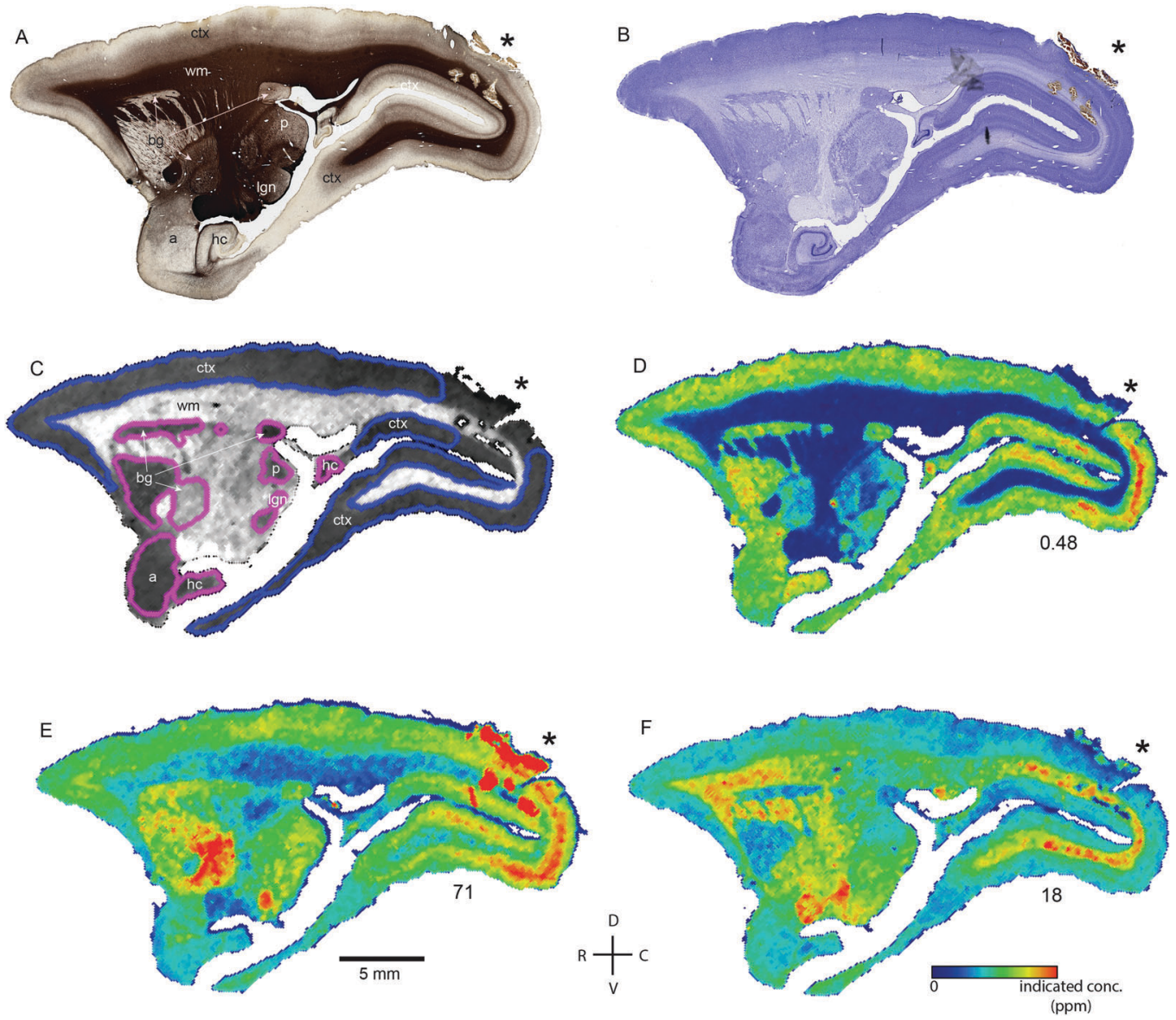

Fig. 1 Histological and metallomic data from a representative para-sagittal section of case CJ166. (A and B) Myelin (A) and Nissl-stained histological sections indicating major anatomical divisions and tissue compartments visible at approximately $4.7 \mathrm{~mm}$ lateral to the interhemispheric midline. Asterisks indicate the site of tissue damage resulting from placement of a linear electrode array during in vivo electrophysiological recording. Metal concentrations in this region were excluded from analysis due to gliosis and extravasated blood near the electrode site. (C) Carbon scan used for demarcation of ROIs used in metallomic analysis. The boundaries of ROls for compartments visible in this section are indicated by purple and blue outlines. (D) False color image of the whole-brain distribution of $\mathrm{Mn}$. Number at lower right indicates the ppm concentration at which the colour scale saturates - individual pixel values may exceed this concentration (peak value of colour scale on lower right). Note low Mn concentration throughout the subcortical white matter. (E) Fe concentration map from the same tissue section shown in (D and E). (F) Zn concentration distribution from the same sections as above. a: amygdala; bg: basal ganglia; Ign: lateral geniculate nucleus; ctx: cerebral cortex; wm: white matter; hc: hippocampus; p: pulvinar.

organized and cytoarchitectonically, histochemically, and developmentally distinct from higher order association cortical areas. ${ }^{29}$ Primary sensory cortical areas include the primary visual (V1), primary auditory (A1) and primary somatosensory (S1) areas, respectively. All of these areas are readily identified histologically using a variety of methods, including Nissl, myelin, and cytochrome oxidase staining. Digitally scanned tissue sections from series adjacent to the LA-ICP-MS scanned sections were aligned and parcellated according to published stereotaxic atlases. ${ }^{30,31}$ The resulting demarcations were exported to ImageJ and used for evaluation of the metal scanning data.

\section{LA-ICP-MS imaging}

Biometal content was assessed using a 8800 triple quadrupole ICP-MS unit (Agilent Technologies, Mulgrave, VIC, Australia) which received vaporized brain tissue in argon carrier gas (flow rate $1.05 \mathrm{~L} \mathrm{~min}^{-1}$ ) from a NWR213 laser ablation system (Kennelec Scientific, Mitcham, VIC, Australia). Acquisition parameters for both instruments are summarized in Table 2. Standards consisting of homogenized lamb brain spiked with known concentrations of each target elemental species were scanned interleaved with the marmoset brain sections. ${ }^{5,29}$ Tissue sections were scanned using a $100 \mu \mathrm{m}$ square laser spot size at a speed of $400 \mu \mathrm{m} \mathrm{s}^{-133}$ and a laser fluency of approximately $0.2-0.4 \mathrm{~J} \mathrm{~cm}^{-2}$ (usually $0.30 \mathrm{~J} \mathrm{~cm}^{-2}$ ). The flat mounted section (case M1598) was scanned using a $50 \mu \mathrm{m}$ laser spot size at $200 \mu \mathrm{m} \mathrm{s}^{-1}$.

Unpublished observations indicate that storage of dried tissue sections does not substantially affect the distribution and content of the metals of interest here. However, freezing and storage in formalin may affect tissue metal concentrations (see, e.g. ref. 32 and 34), and caution is therefore warranted when comparing metal concentrations across tissues prepared using different protocols.

\section{Quantification and image processing}

Signal intensities (counts per seconds, CPS) of the tissue standards were analysed in iolite. ${ }^{35}$ The derived correction 
Table 2 Operational parameters of the LA-ICP-MS experiments. NB: this information is reported in standardised format across multiple publications

8800 triple quadrupole ICP-MS

Agilent Technologies (Mulgrave, VIC, Australia)

\begin{tabular}{ll}
\hline Carrier gas, $\mathrm{L} \mathrm{min}^{-1}$ & Argon, 1.05 \\
Scan mode & Peak hopping \\
Sampler/skimmer cone & Pt, Ni base \\
Collision gas, mL min & $\mathrm{H}_{2}, 3$ \\
Monitored isotopes & ${ }^{13} \mathrm{C} ;{ }^{31} \mathrm{P} ;{ }^{55} \mathrm{Mn} ;{ }^{56} \mathrm{Fe} ;{ }^{63} \mathrm{Cu} ;{ }^{66} \mathrm{Zn}$ \\
Dwell time/mass, ms & 38.4
\end{tabular}

New Wave Research 213 laser ablation system ESI Kennelec Scientific (Mitcham, VIC, Australia)

\begin{tabular}{ll}
\hline Ablation mode & Line mode \\
Laser wavelength, $\mathrm{nm}$ & 213 \\
Laser energy density, $\mathrm{J} \mathrm{cm}^{-2}$ & $0.2-0.3$ \\
Repetition frequency, $\mathrm{Hz}$ & 20 \\
Laser shape & Square \\
Laser spot size, $\mu \mathrm{m}^{2}$ & 10000 \\
Line spacing, $\mu \mathrm{m}$ & Laser edge length, 100 \\
Laser scan speed, $\mu \mathrm{m} \mathrm{s}^{-1}$ & $4 \times$ laser edge length, 400
\end{tabular}

factors were used to account for drift in data sampling specifics over time and for computing the parts-per-million (ppm) concentration from the CPS data of each target element using Matlab (R2015b, Mathworks Inc., Natick, MA). The corrected and converted data was concatenated across tissue slides and saved as text files using Biolite software. ${ }^{7}$ Text images were then imported into ImageJ (NIH, Bethesda, MD), where background noise was removed and the data was median filtered (1 pixel). Carbon-13 $\left({ }^{13} \mathrm{C}\right)$ images were used for alignment with histological sections and anatomical atlases of the marmoset brain ${ }^{30,31}$ and identification of target regions of interest (ROI) containing the major brain tissue types. The resulting ROIs were copied to the managnese, iron, and zinc scans, and the pixel values for each element within each ROI were saved as CSV data files for quantitative analysis. Images for figures were prepared in Image and Adobe Photoshop and Illustrator (CS6, Adobe Systems, Mountain View, CA). Display images were resized, cropped, and adjusted for brightness, contrast, and level, and non-tissue backgrounds were removed. However, histological artefacts, incidental noise, and tissue irregularities which impinged on the sample area were preserved in the final images. All adjustments were applied equally across the tissue area.

\section{Statistical analysis}

For purposes of comparison across anatomical areas, each LA-ICP-MS scanned section was parcellated into ROIs as described above, and the mean concentration of each target metal was computed from the individual pixel values within the ROI. This value was obtained from each tissue section which contained the anatomical structure of interest, and the resulting observations from each animal were pooled into a single dataset. Nonparametric methods including the Kruskal Wallace test and Mann-Whitney $U$ test were used to assess the relative metal concentrations of each anatomical area studied. Statistically significant differences were identified using a conservative alpha level of 0.01 , which reflects the large differences in volume of the respective tissue compartments (i.e. white matter and cortex were evident in virtually every studied section, while smaller structures such as the septum and substantia nigra were only present in a few sections from each animal), and decreases the probability of a false discovery. ${ }^{36}$ Statistical calculations were performed and graphs/charts were prepared using Prism software (v.6.0h; GraphPad Software, La Jolla, CA).
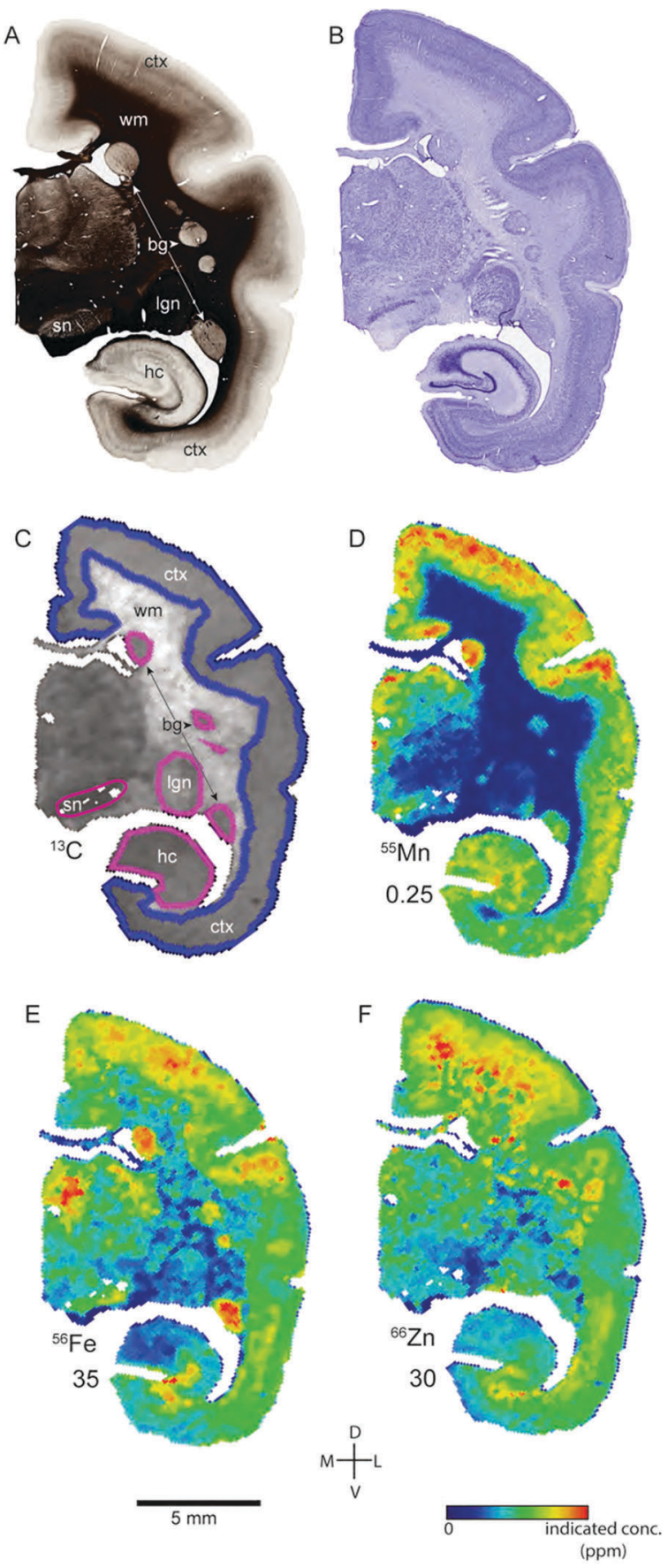

Fig. 2 Histological and metallomic data from a representative coronal section of case CJ154 (approximate location: $5.6 \mathrm{~mm}$ anterior from the interaural line). Figure conventions as indicated in Fig. 1. sn: substantia nigra. All other abbreviations as in Fig. 1. 
All data were stored on the Open Science Framework platform (Center for Open Science, Charlottesville, VA; http://www.osf.io).

\section{Results}

The distribution of each of the transition metals assessed in this study exhibited regional variations which were consistent with anatomical structures as defined by cyto-, myelo-, and chemo-architectonic analyses, with the exception of copper. Copper scanning yielded frequent gradient artefacts along the outer edge of the brain tissue, which extended inward, often to the white matter boundary. The source of this artefact is currently under investigation, but copper data were not included in the final analysis for this report. Thus, all subsequent descriptions will be confined to manganese, iron, and zinc.

Transition metal concentrations were not homogenously distributed across brain structures, as shown in Fig. 1 and 2 . Among the gross patterns observed in this study were sharp differentiation of white matter from the cerebral cortex and major subcortical nuclei by all three metal species. This pattern was most evident in the Mn and $\mathrm{Zn}$ scans, with high Mn levels evident in the grey matter and virtually absent from white matter (Fig. 1D and 2D), and higher $\mathrm{Zn}$ concentrations in the white matter (Fig. 1F and 2F). An intermediate pattern was observed in the Fe scan, with grey matter containing somewhat higher concentrations of Fe than white matter (Fig. 1E and 2E).

\section{Manganese}

Regions of highest $\mathrm{Mn}$ concentration, with respect to both mean and peak values, included the amygdala, basal ganglia, cortex, and hippocampus. In contrast, the claustrum and lateral geniculate nucleus exhibited the lowest concentrations among the grey matter regions studied (Table 3 and Fig. 3A-C). As indicated above, the white matter compartment exhibited strikingly lower Mn concentrations than grey matter across all nuclei studied, and white- and grey-matter boundaries were clearly resolved in $\mathrm{Mn}$ images. Pairwise comparison of the mean concentration obtained for each tissue compartment using the Kruskal-Wallis test indicated significant differences between the white matter and each grey matter compartment tested
(Table 3). Notably, Mn levels in the smaller volume nuclei including the superior colliculus, substantia nigra, and septum, were similar to the levels found in the larger compartments such as the cortex and basal ganglia. These smaller compartments were often the sites of greatest contrast with Fe, in terms of relative concentration.

\section{Iron}

In general, Fe was the most prevalent transition metal observed in this study, with most grey matter compartments containing 20-30 ppm of non-heme Fe. The grey matter compartments with the highest mean Fe concentration was the substantia nigra (approximately $40 \mathrm{ppm}$ ), which is consistent with results from rodent, ovine, and primate studies. Similar mean $\mathrm{Fe}$ concentrations were observed in the pulvinar and basal ganglia (especially the globus pallidus). Moderate Fe levels were observed in all of the grey matter compartments studied, with significantly lower Fe concentration in the white matter. In addition, pairwise comparison of the individual compartments indicate significant differences between the basal ganglia and amygdala, cortex, claustrum, and hippocampus; and between the hippocampus and the pulvinar and substantia nigra, in addition to the white matter contrasts (Table 3).

\section{Zinc}

The most striking feature of the pattern of $\mathrm{Zn}$ distribution in the marmoset brain was the near parity of mean $\mathrm{Zn}$ concentration between the grey and white matter compartments. This resulted in a much higher relative $\mathrm{Zn}$ level in the white matter compared with either Mn or Fe. Moreover, initial examination of the $\mathrm{Zn}$ scans suggests that $\mathrm{Zn}$ is inhomogeneously distributed even within the white matter compartment, with certain tracts, including the anterior commisure, corpous callosum, and corona radiata exhibiting locally high levels of $\mathrm{Zn}$. This possibility will be explored quantitatively in a larger sample in future studies. It is also noteworthy that the overall pattern of $\mathrm{Zn}$ distribution in 3 out of 4 animals suggests that $\mathrm{Zn}$ concentration demarcates the the white matter-grey matter boundary quite clearly, with higher relative $\mathrm{Zn}$ levels apparent in the white matter. In one case (F1510), this pattern was reversed. The pulvinar nucleus of the thalamus also exhibited relatively low $\mathrm{Zn}$ concentrations, with

Table 3 Results of pairwise comparisons from the Kruskal-Wallis test for comparison of manganese, iron, and zinc concentrations across marmoset brain areas. Alpha was set at $p=0.01$ and Dunn's test was employed to correct for multiple comparisons. Exact $p$ values were computed in GraphPad Prism for each comparison. Significant results are indicated by the chemical symbols in the matrix for each comparison, and non-significant values are indicated by a (-) sign

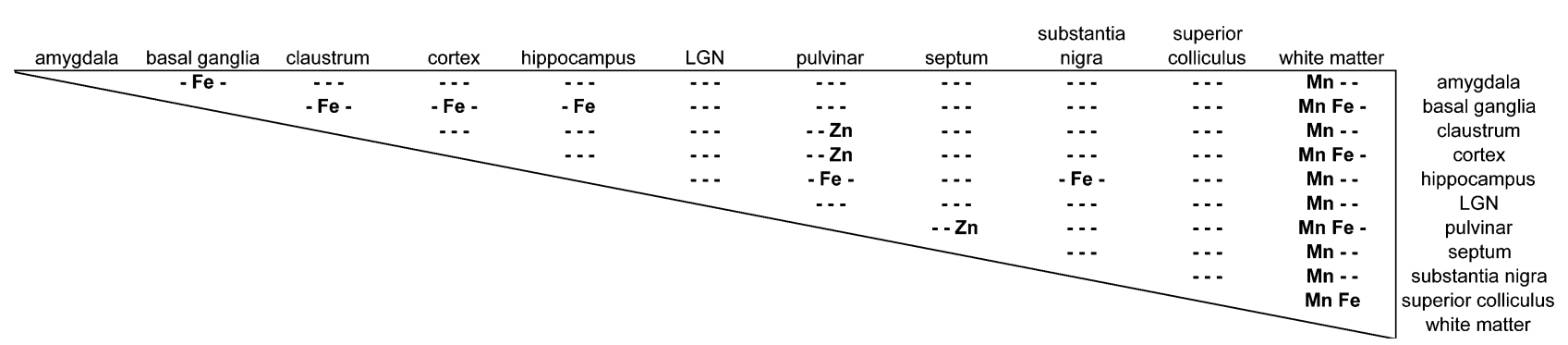

Chemical symbols indicate positions in matrix where $p<0.01$ for difference in mean concentration between compartments, $(-)$ indicates $p>0.01$. 

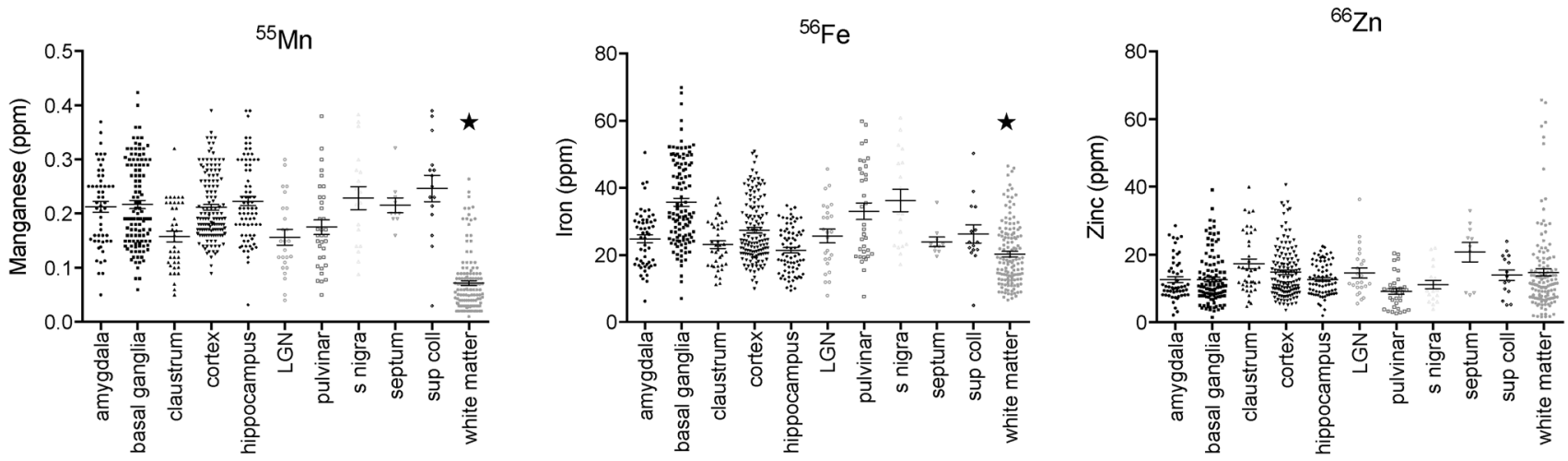

Fig. 3 Mean metal concentration across all ROI for each anatomical division and tissue compartment. Scatter plot includes all tissue sections from each case in which the respective ROI was identified (all except case M1598). Data were pooled across animals: asterisks indicate significant ( $p$ 《 0.01 ) differences between the subcortical white matter and all other divisions. Horizontal bars indicate mean \pm SEM for each section ROI mean value examined. Note unequal $y$-axis scales between metals.
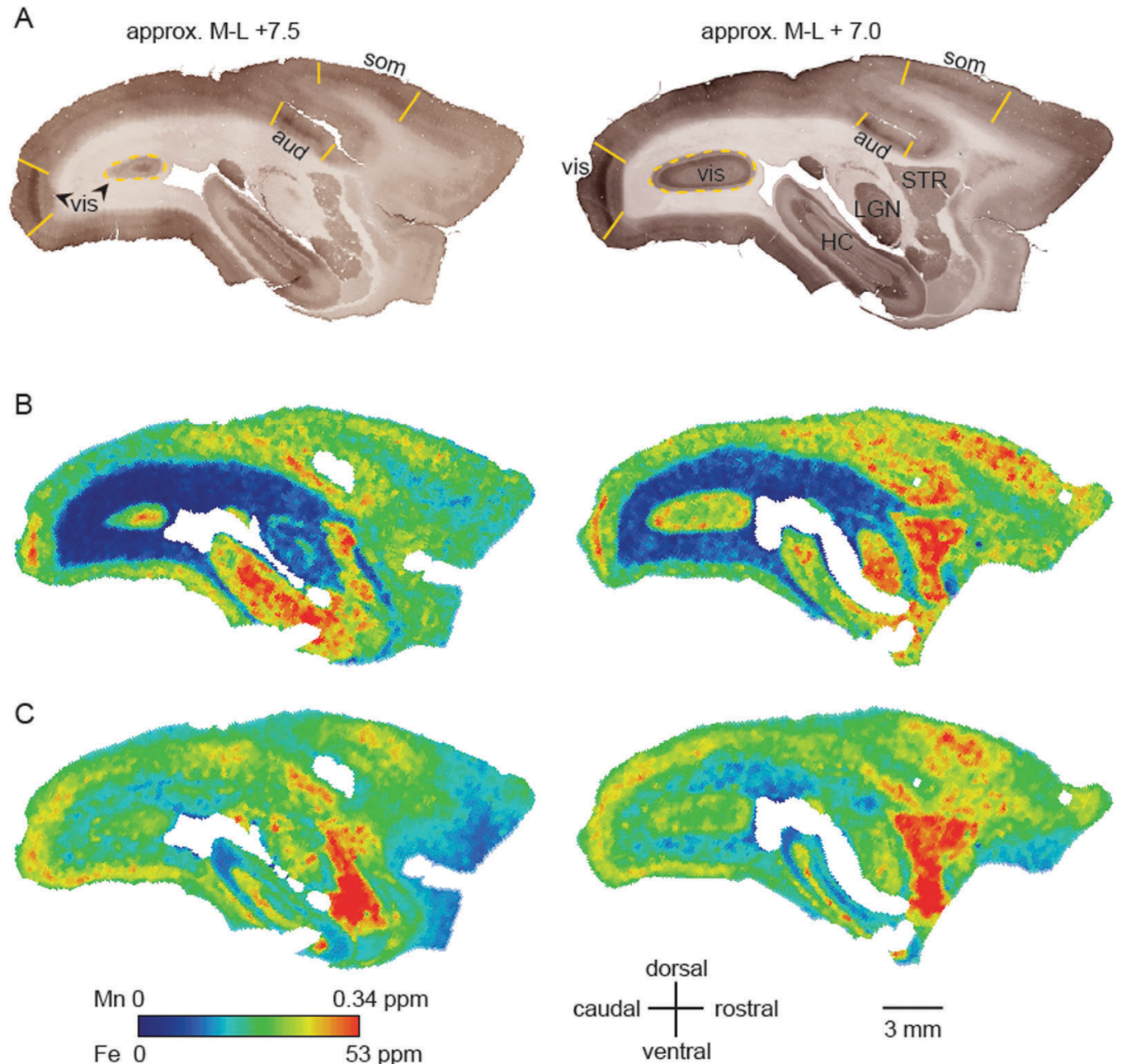

Fig. 4 High relative $\mathrm{Mn}$ and Fe concentrations coincide with three primary sensory areas. Cerebral sections of an adult common marmoset were cut in the sagittal plane at approximately +7.5 (left) and $+7 \mathrm{~mm}$ (right) in the medio-lateral axis. (A) Sections histochemically stained for cytochrome oxidase (CO). Anatomical features of particular relevance are the primary visual cortex (vis), the primary auditory cortex (aud) and the somatosensory cortex (som) which are indicated and readily identifiable by intense $\mathrm{CO}$ staining. Additional prominent structures are identified in the right image. (B and C) False color images with signal intensity for $\mathrm{Mn}(\mathrm{B})$ and $\mathrm{Fe}(\mathrm{C})$, as specified on the color scale. Abbreviations: HC: hippocampus, LGN: lateral geniculate nucleus, STR: striatum. 
pairwise comparison indicating significantly higher $\mathrm{Zn}$ in the claustrum, cortex, and septum, relative to the pulvinar (Table 3).

\section{Metallomic profile of specific brain compartments}

Within-compartment variation of metal concentration was assessed in the cerebral cortex, amygdaloid complex, and hippocampus. These areas were selected as they have clearly identifiable anatomical and subdivisions, allowing for comparison of metal concentration differences against structural and functional characteristics. Substantial variation was apparent across cytoarchitectonically defined areas of the cerebral cortex, with enrichment of both $\mathrm{Mn}$ and Fe specifically noted in the primary sensory areas.

Primary sensory areas. Both $\mathrm{Mn}$ and Fe exhibited elevated concentrations in primary sensory areas, including visual, somatosensory, and auditory areas. This pattern was evident in both the sagittal (Fig. 4) and coronal (Fig. 5) planes. In primary visual cortex (V1), the areas of high concentration were mainly restricted to the middle cortical laminae, with Mn appearing more confined in the superficial-to-deep axis than Fe (Fig. 4 and 5). A similar pattern was also evident in the primary auditory cortex (Fig. 4), while the region of increased metal concentration extended into the supragranular and infragranular layers in somatosensory cortex. ${ }^{4}$ To visualize the co-localization of relative $\mathrm{Mn}$ and Fe concentrations, we displayed each as intensity of one color and merged the data into one image (Fig. 6). This analysis demonstrated that high $\mathrm{Mn}$ and Fe concentrations were evident in the middle cortical laminae of the primary visual and auditory cortex. Importantly, Mn and Fe are differentially distributed in other regions of the brain, e.g. basal ganglia, hippocampus, and the white matter, indicating that they are not simply co-localized throughout the marmoset brain.

We compared the pattern of $\mathrm{Mn}$ and Fe distribution in V1 to the well-characterized 'blob' and 'interblob' pattern of CO heterogeneity, ${ }^{37}$ using flat-mounted sections from the supragranular layers for LA-ICP-MS analysis. Fig. 5A illustrates the generation of the flat mount according to established procedures. ${ }^{29} \mathrm{CO}$ staining in V1 clearly showed 'blobs' in supragranular layer (Fig. 7B upper inset). More intense but homogeneous CO staining was observed in the infragranular layer (Fig. 7B lower inset), presumably layer IV. Sections were not cut perfectly parallel to the cortical surface with deeper layers being present in the top left corner of the sections showing an intense and homogeneous CO band (Fig. 7B). To assess the distribution of metal content, we examined a section immediately adjacent the $\mathrm{CO}$ stained section shown in Fig. 7B. High Mn concentration was not evident in the supragranular layers of V1. However, elevated Mn concentrations were present in the infragranular cortical layers, as evident in the top left corner of the flattened cortical section.

The Fe concentration was also selectively elevated in V1. We attempted to relate this observation to the known chemoarchitectonic organization of primate V1 by comparing the $\mathrm{Fe}$ distribution to the 'blob' and interblob pattern of cytochrome oxidase labelling. Following manual and automated definitions
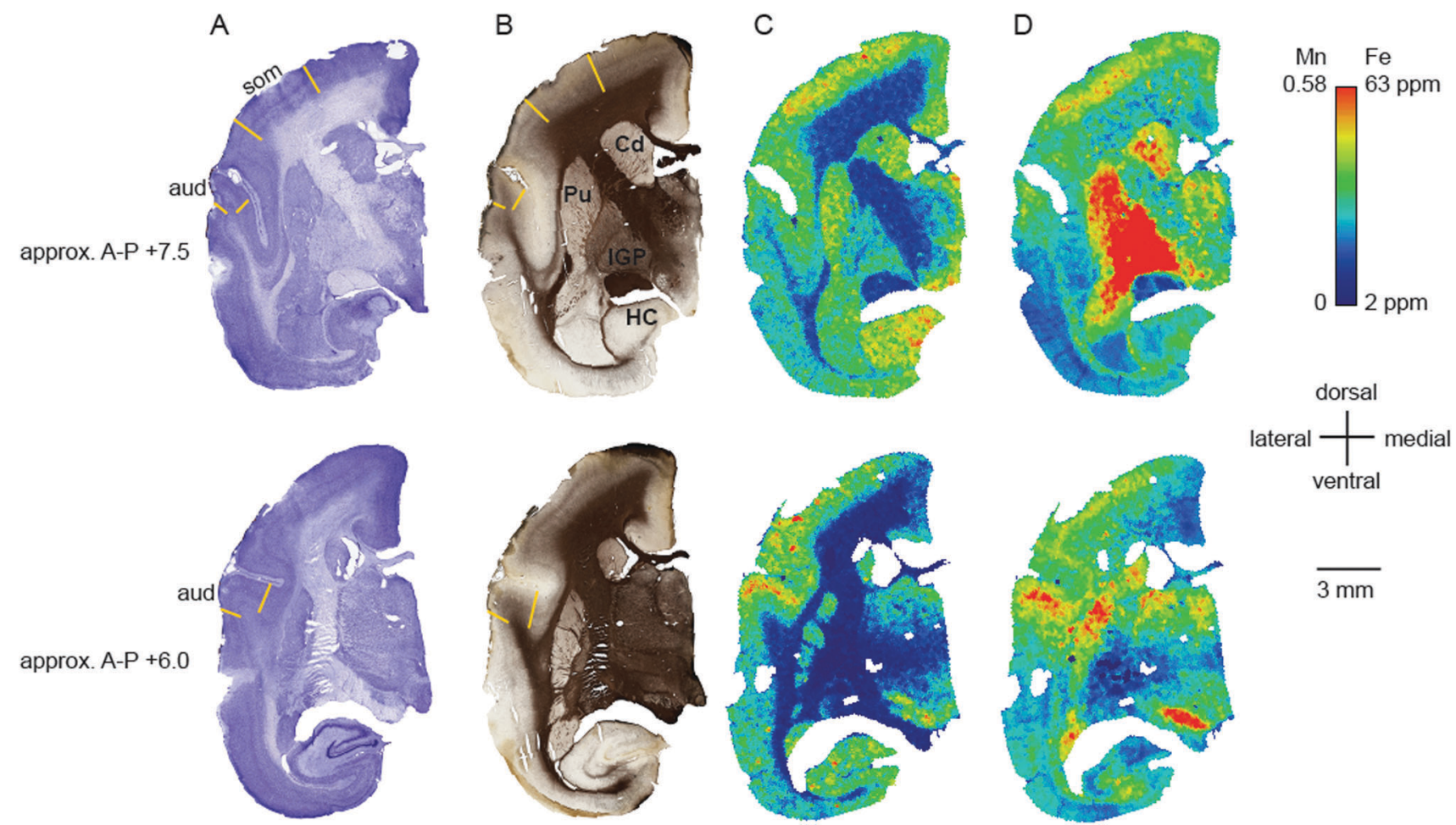

Fig. 5 High relative $\mathrm{Mn}$ and Fe concentrations coincide with two primary sensory areas. Cerebral sections of an adult common marmoset were cut in the coronal plane at approximately +7.5 (top) and $+6 \mathrm{~mm}$ (bottom) in the anterior-posterior axis. (A) Nissl stained sections. The primary auditory (aud) and somatosensory (som) cortical areas are indicated and labelled. (B) Adjacent sections stained for myelin. The respective cortical areas are indicated as well. Additional prominent structures are identified in the top image. ( $C$ and D) False color images where the colors indicate the signal intensity for $\mathrm{Mn}$ (C) and Fe (D), as specified on the color scale. Abbreviations: Cd: caudate nucleus, HC: hippocampus, IGP: internal globus pallidus, Pu: putamen. 
A approx. $M-L+11$
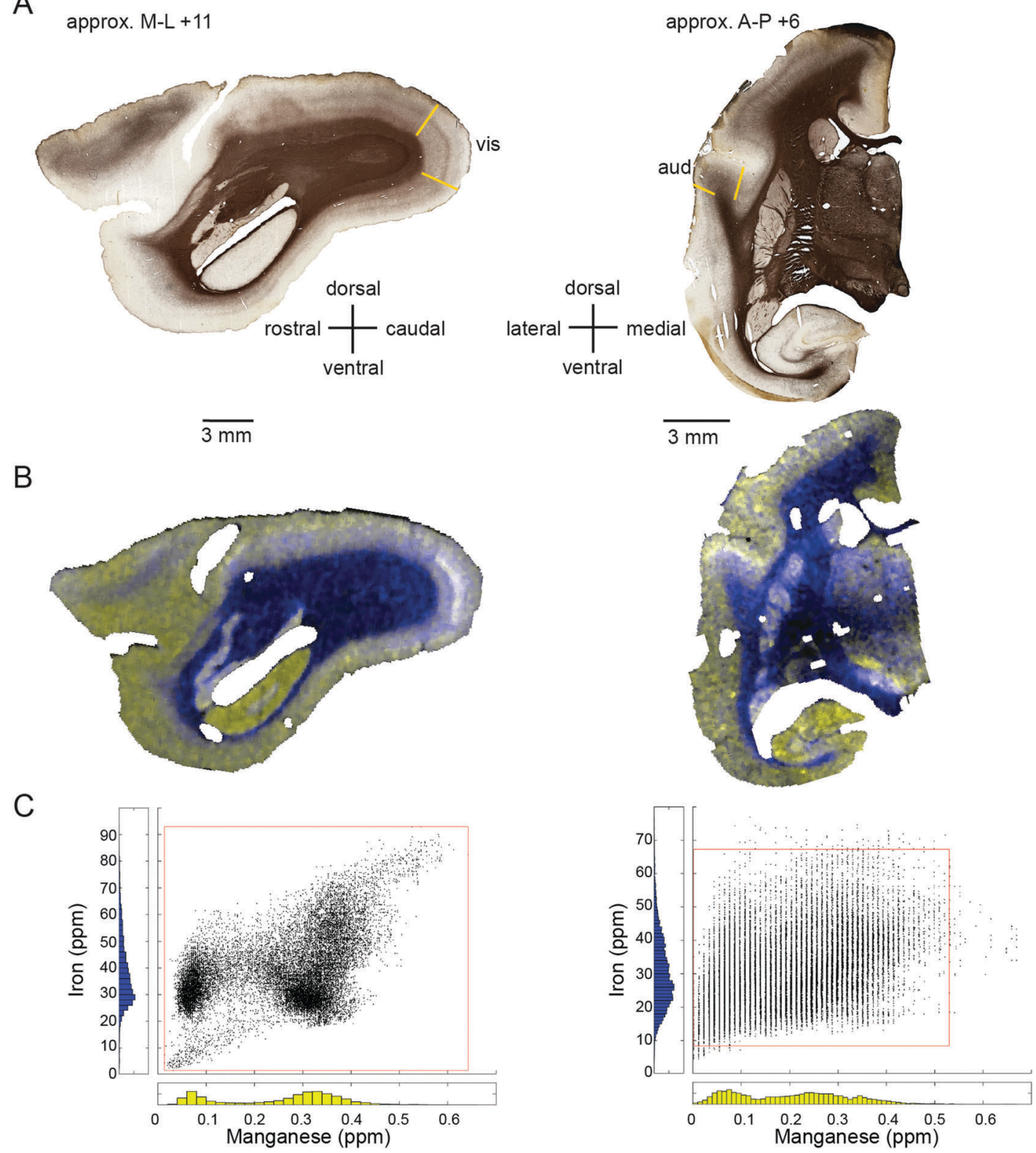

Fig. 6 Co-localization of relatively high signal intensities in the Mn and Fe data displayed in a sagittal (left) and coronal section (right). (A) The myelin stained sections indicate the primary visual cortex (vis) and the primary auditory cortex (aud). (B) The merged image of the Mn and Fe signal intensities. White areas correspond to the co-localization of both high $\mathrm{Mn}$ and high Fe signal. Grey and black areas correspond to the co-localization intermediate and low signal intensities, respectively, in both $\mathrm{Mn}$ and Fe. Yellow and blue areas correspond to the dominance of either the Mn or Fe signal intensity, respectively. (C) Scatter plots of the Mn ( $x$-axis) and Fe ( $y$-axis) signal intensities are shown. For the sagittal section, all data was included. For the coronal section the signal intensities were clipped to the 0.25 th and 99.75 th percentile (red square) to eliminate the effect of outliers, particularly in the Mn data. Data points along the diagonal yield black over grey to white colors in the merged image and indicate equally low, medium and high relative concentrations in both $\mathrm{Mn}$ and Fe, respectively. Data points above and below the diagonal yield blue and yellow colors in the merged image and indicate higher relative intensity in the Fe or the $\mathrm{Mn}$ signal, respectively.

of blobs, and a spatial fast Fourier transform, which unequivocally identified 'blobs' in the CO stain, similar image analysis algorithms were applied to the Mn and Fe data. No evidence of 'blob' architecture was detected in the distributions of either metal (Fig. 7C insets).

\section{Hippocampus and amygdala}

Localisation of Mn and Fe was also evident within the hippocampus (Fig. 8A-L). We observed local enrichment of $\mathrm{Mn}$ in the CA3 region and dentate gyrus of the hippocampus, and in the nearby entorhinal cortex (Fig. 8D and J). In contrast, local Fe enrichment was evident in the CA1 region and in the subiculum, with a relative paucity of $\mathrm{Fe}$ in the $\mathrm{CA} 3$ and dentate gyrus (Fig. $8 \mathrm{E}$ and $\mathrm{K}$ ). Relatively little $\mathrm{Zn}$ was observed in the hippocampus, although high levels were evident in the white matter underlying the adjacent subiculum and entorhinal cortex (Fig. $8 \mathrm{~F}$ and $\mathrm{L}$ ).

In contrast to the localisation of metals within subdivisions of the hippocampus, no systematic variation of metal concentration 

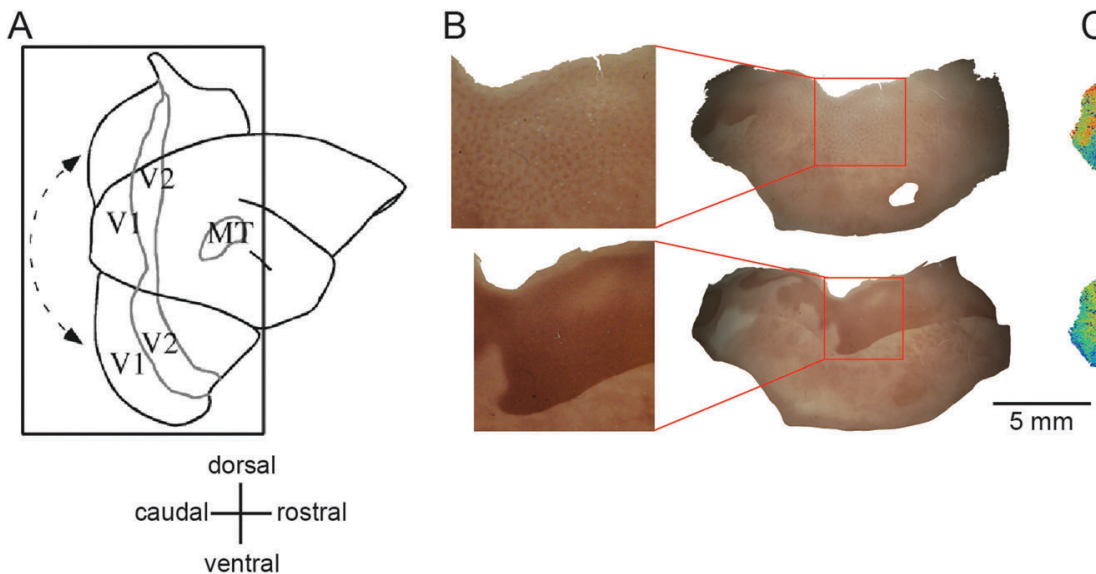

C

Fig. 7 Generation of the flat mount, selection of the section for laser ablation-inductively coupled plasma-mass spectrometry and its results. (A) Illustration of the generation of a flat mount of the primary visual cortex is displayed (sketch adapted from Rosa et al., 2005, with permission from the authors). ${ }^{46}$ The image of the flat mount has to be rotated $90^{\circ}$ clockwise to represent the orientation of the cytochrome oxidase (CO) stained sections in $\mathrm{B}$ and the laser ablated sections in (C). (B) Images of the CO stained section \# 1 (top) and \# 6 (bottom). Note that, despite clearly exhibiting blobs in V1 (blow-up), the overall CO staining intensity is low in section one. The tissue was cut not perfectly parallel to the cortical surface. Hence the intense but homogeneous band of CO staining, presumably layer IV, starts to emerge in the top left corner of section \# 1. (C) To test for the existence of spatial patterns resembling the $\mathrm{CO}$ 'blob-interblob' arrangement, we investigated the distribution of $\mathrm{Mn}$ (top) and Fe (bottom) concentrations in the section adjacent to $\mathrm{CO}$ stained section \# 1. The blow-up shows the $\mathrm{Mn}$ and Fe distribution in the area where 'blob' resembling arrangement of concentrations was to be expected.

was evident between the lateral, basolateral, or basomedial subdivisions of the amygdaloid complex, although each metal was present to some extent in the amygdala (Fig. 9).

\section{Comparison of metal levels across species}

The mean brain tissue concentrations of $\mathrm{Mn}, \mathrm{Fe}$, and $\mathrm{Zn}$ obtained by LA-ICP-MS were generally consistent with reported values for other mammalian species, as shown in Table 4. Fixed sections of marmoset brain were slightly higher in $\mathrm{Zn}$ and $\mathrm{Mn}$ than rodent or ovine species, and Fe level in the marmoset were comparable to those reported in rats and mice, especially in high-Fe regions such as the substantia nigra and basal ganglia. One notable feature of this study was the disparity between observed Mn concentrations and levels reported for human autopsy specimens, ${ }^{38}$ which contained approximately 10 times as much $\mathrm{Mn}$ as the postmortem marmoset brain (Table 4).

\section{Discussion}

The principal objective of this study was to establish baseline levels of transition metals across tissue types in the non-human primate brain, with the eventual goal of generating a wholebrain atlas of biometals. The marmoset represents an ideal model species for this effort, due to its small size, relative ease of handling and husbandry, and physiological similarity to humans. Most importantly for our purposes, the marmoset brain is small (approximately $3 \mathrm{~cm}$ along the rostral-caudal axis), which allows for LA-ICP-MS scanning of an entire brain, with accurate registration and identification of anatomical structures via histological staining. Given current constraints on scanning time and laser performance, it is not feasible to scan a full brain from a larger primate at similar spatial resolutions.

Imaging of coronal and parasagittal sections at $200 \mu \mathrm{m}$ intervals yielded concentration maps of ${ }^{13} \mathrm{C},{ }^{55} \mathrm{Mn},{ }^{56} \mathrm{Fe}$, and ${ }^{66} \mathrm{Zn}$ at sufficient spatial resolution to identify major anatomical features and to differentiate brain tissue compartments, including grey- and white-matter boundaries, ventricles, and major blood vessels. High resolution imaging of histological stains from adjacent tissue series allowed for precise alignment of images and reliable measurement of metal concentrations within each compartment.

The most consistent differentiator of white matter $v s$. grey matter in the marmoset brain was Mn, which was present in uniformly low concentrations in the white matter relative to all other compartments. In three out of four cases in which it was assessed, Zn was also useful for delineation of the white matter boundary, with higher relative concentrations in the white matter. This pattern is somewhat confusing in light of the fourth case, which showed the opposite pattern (i.e. lower $\mathrm{Zn}$ in white matter vs. grey). One possible explanation for this discrepancy may be equipment-related, as it was necessary to perform a scheduled maintenance procedure on the laser and to synthesise new reference standard materials during the study period (which also accounts for the long delay in scanning some of the test material). The aberrant case in this study (F1510) was scanned very early in the study period (prior to the equipment refit), while all of the other scans were performed afterwards. We are thus inclined to believe that the Zn concentration ratio of white $v s$. grey matter is higher than the ratios of other tested metals, but acknowledge that this explanation is tentative at present, especially since it was only the $\mathrm{Zn}$ readings which differed in the gross pattern of compartmentalization before and after servicing of the equipment. 


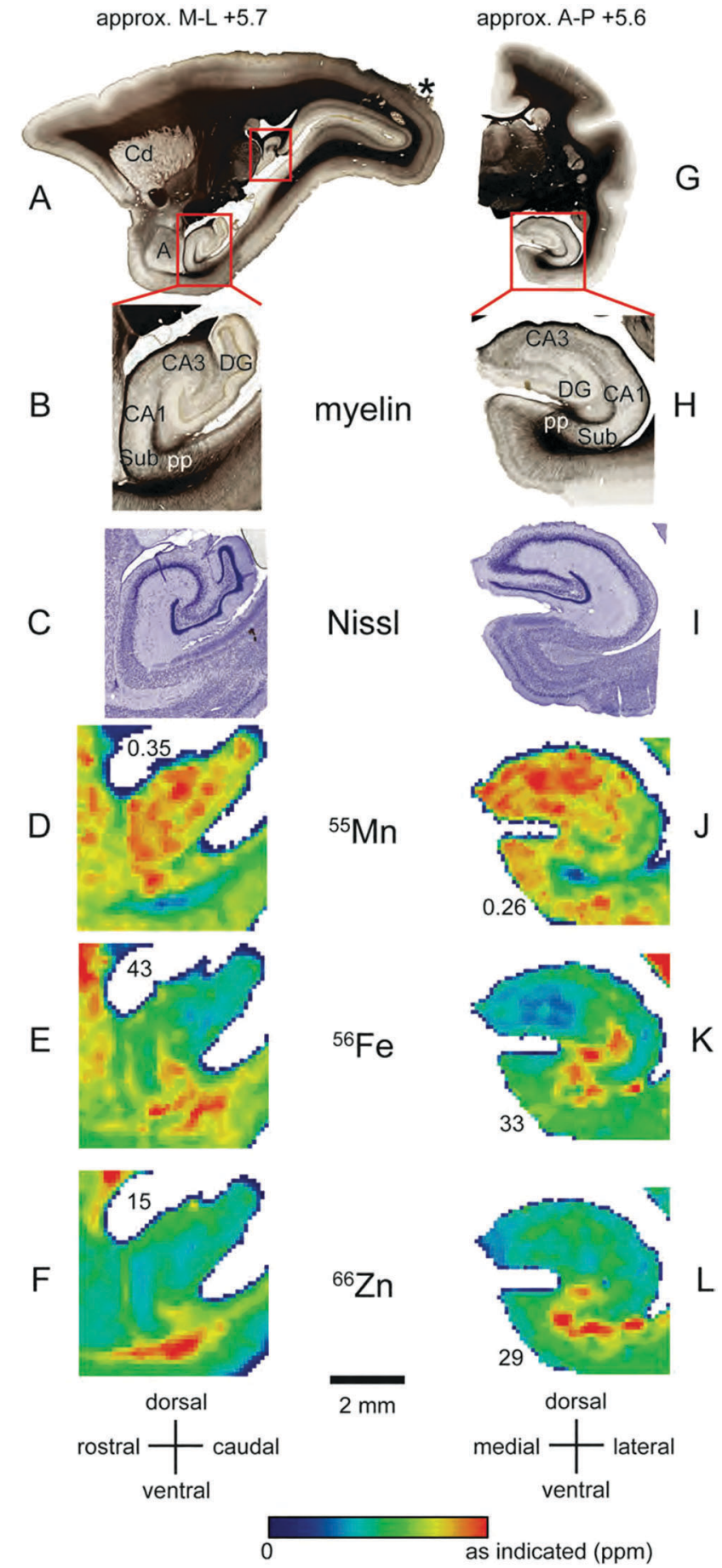

Fig. 8 Iron, manganese and zinc concentrations in the hippocampus. The top row (A and $G$ ) shows myelin stained sections cut in the sagittal (A) or coronal (G) plane at approximately $+5.7 \mathrm{~mm}$ in the medio-lateral axis and $+5.6 \mathrm{~mm}$ in the anterior-posterior axis, respectively. $(B$ and $H$ ) Enlarged view of insets in ( $A$ and $G$ ) indicating main hippocampal divisions and other anatomical areas examined. In the sagittal plane (left) only the ventral, but not the dorsal hippocampus (additional red box) is highlighted. ( $C$ and I) Nissl stained hippocampal sections from the insets in (A and G), respectively. (D-F and J-L): False color image of LA-ICP-MS analysis from a single section showing concentrations of $\mathrm{Mn}(\mathrm{D}$ and $\mathrm{J}), \mathrm{Fe}(\mathrm{E}$ and $\mathrm{K})$ and $\mathrm{Zn}(\mathrm{F}$ and $\mathrm{L})$ in the hippocampal proper whereby values are indicated as in Fig. 1. Abbreviations: a: amygdala, CA1,3: cornu ammonis regions, Cd: caudate nucleus, DG: dentate gyrus, Sub: subiculum, pp: performant path. The scale bar applies to all zoomed images.

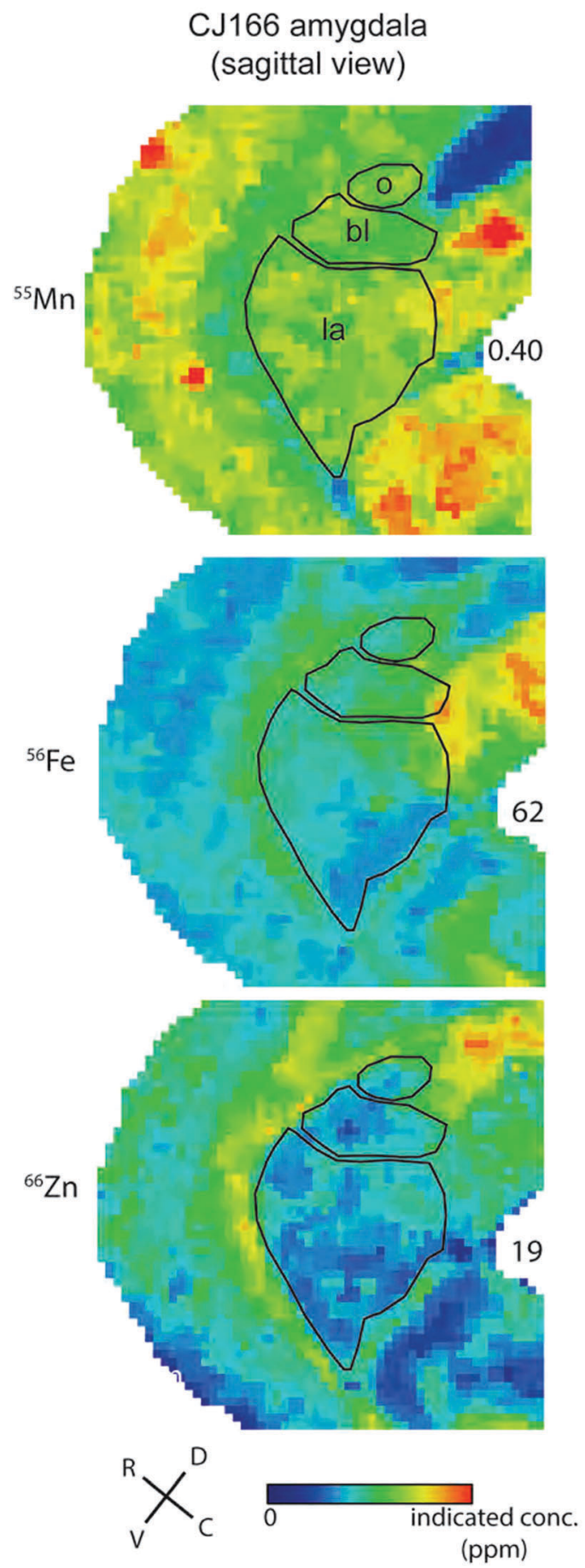

Fig. 9 Para-sagittal metallomic map of the amygdala approximately $6.4 \mathrm{~mm}$ lateral to the interhemispheric midline. Upper, mid, and lower panels show relatively homogeneous distribution of $\mathrm{Mn}$, Fe, and $\mathrm{Zn}$, respectively, within subdivisions of the marmoset amygdala. Abbreviations in top panel: la - lateral division of the amygdala; $\mathrm{bl}$ - basolateral division of the amygdala; o - other, unspecified divisions of the amygdala (not included in quantitative analysis). Not shown in this section: basomedial division of the amygdala.

Metallomic scanning of flat-mounted sections of the occipital cortex showed spatial overlap between regions of high Fe concentration and the primary visual cortex, but the characteristic CO 'blobs' were not observed in the Fe distribution. The comparative 
Table 4 Comparison of marmoset tissue metal concentrations with published values from other species. This table represents an overview of available information in which concentrations were reported as parts per million or in a form easily converted to ppm. It is not intended as a comprehensive survey of the metallomic literature, as numerous studies report values as counts per second or in other units not easily comparable to our data

\begin{tabular}{|c|c|c|c|c|c|c|c|}
\hline Species & Method & Brain region & Mn & $\mathrm{Fe}$ & $\mathrm{Zn}$ & Notes & Ref. \\
\hline \multirow[t]{3}{*}{ Sheep } & \multirow{3}{*}{$\begin{array}{l}\text { Atomic absorption } \\
\text { spectrophotometry } \\
\text { (AAS) }\end{array}$} & Cortex & $0.29-0.61$ & $18.17-34.17$ & $1.23-4.28$ & \multirow[t]{3}{*}{$\begin{array}{l}\text { Juvenile animals cortex values } \\
\text { across all subdivisions }\end{array}$} & \multirow[t]{3}{*}{ Bakirdere et al. $(2011)^{41}$} \\
\hline & & Hippocampus & 0.35 & 13.77 & 2.72 & & \\
\hline & & Thalamus & 0.36 & 17.38 & 2.41 & & \\
\hline \multirow[t]{2}{*}{ Macaque } & \multirow[t]{2}{*}{ AAS } & Cortex & 0.25 & na & na & \multirow[t]{2}{*}{ Frontal cortex only } & \multirow[t]{2}{*}{ Dorman et al. $(2006)^{42}$} \\
\hline & & Basal gangila & $0.34-0.48$ & na & na & & \\
\hline \multirow[t]{5}{*}{ Human } & \multirow[t]{5}{*}{ AAS } & Cortex & $1.13-1.69$ & na & na & \multirow{5}{*}{$\begin{array}{l}\text { Autopsy samples } \\
\text { Range across all subdivisions }\end{array}$} & \multirow[t]{5}{*}{ Bonilla et al. $(1982)^{38}$} \\
\hline & & Basal ganglia & $1.29-1.89$ & na & na & & \\
\hline & & Substantia nigra & 1.02 & na & na & & \\
\hline & & Thalamus & 0.94 & na & na & & \\
\hline & & Superior colliculus & 1.21 & na & na & & \\
\hline \multirow[t]{4}{*}{ Human } & \multirow[t]{4}{*}{ ICP-MS } & Cortex & $1.0-1.2$ & na & $55-68$ & Forensic autopsy samples & \multirow[t]{4}{*}{ Ramos et al. $(2014)^{43}$} \\
\hline & & Basal ganglia & $2.0-2.5$ & na & $45-61$ & Ranges across all subdivisions & \\
\hline & & Hippocampus & 1.20 & na & 70 & & \\
\hline & & Whole brain average & 1.30 & na & 53 & & \\
\hline Mouse & STIM/PIXE & Whole brain & na & 41.3 & 20.1 & $\begin{array}{l}\text { Unspecified ROI across } \\
\text { brain areas }\end{array}$ & Rajendran et al. $(2009)^{45}$ \\
\hline \multirow[t]{3}{*}{ Mouse } & \multirow[t]{3}{*}{ LA-ICP-MS } & Cortex & na & $6.0-10.0$ & 5.0 & \multirow[t]{3}{*}{$\begin{array}{l}\text { Estimated from Fig. } 3 \text { and } 4 \\
\text { graphs }\end{array}$} & \multirow[t]{3}{*}{ Portbury et al. $(2016)^{44}$} \\
\hline & & Basal ganglia & na & $5.0-6.0$ & 4.0 & & \\
\hline & & Hippocampus & na & $4.0-5.0$ & 4.5 & & \\
\hline \multirow[t]{6}{*}{ Marmoset } & \multirow[t]{6}{*}{ LA-ICP-MS } & Cortex & 0.20 & 26.73 & 15.91 & \multirow[t]{6}{*}{$\begin{array}{l}\text { Mean values across } \\
\text { tissue sections }\end{array}$} & \multirow[t]{6}{*}{ Current report } \\
\hline & & Basal ganglia & 0.21 & 33.91 & 14.7 & & \\
\hline & & Hippocampus & 0.22 & 20.77 & 13.53 & & \\
\hline & & Thalamus & 0.15 & 27.52 & 14.62 & & \\
\hline & & Substantia nigra & 0.20 & 33.77 & 15.41 & & \\
\hline & & Superior colliculus & 0.20 & 19.91 & 16.54 & & \\
\hline
\end{tabular}

anatomical and functional implications of the observed patterns of metal distribution are considered in detail below.

The marked regional localisation of metals within some tissue compartments (e.g. hippocampus and primary sensory areas of cortex) strongly suggests that variations in metal concentration are associate with the functional properties of brain tissue, as opposed to simple structural or anatomical differences across tissue compartments.

In the cerebral cortex, there was a marked enhancement of $\mathrm{Mn}$ and Fe concentration in the primary sensory areas (those areas which receive the predominant ascending thalamic input for the respective sensory modality). Primary sensory areas have well-characterized distinguishing features with respect to cyto-, myelo-, and chemo-architecture, so it is perhaps not surprising that these regions might be differentiated on the basis of metal content. We initially suspected that the apparent overlap between peaks and troughs of metal concentration would correspond to the characteristic spatial pattern of oscillating high and low CO density in V1, which has been described as a 'blob-interblob' pattern. However, high resolution (50 $\mu \mathrm{m}$ laser spot edge) LA-ICP-MS scanning of flat mounted sections corresponding to the depth of the CO blobs did not reflect the blob structure. Elevated Fe and Mn concentrations were evident in
V1, but the band of maximum density for both metals appeared to be deeper than the blob depth.

High regional $\mathrm{Mn}$ and Fe concentration was also evident in the primary sensory areas of the auditory and visual systems. All of these areas (A1, V1, and S1) exhibit elevated CO staining, indicating a high baseline metabolic profile, but CO is not the only cortical enzyme which is dense in metabolically active areas. The type 2 super oxide dismutase (SOD2) protein is also elevated in regions of high free radical production, which include the primary sensory areas. SOD2 is known to be enriched in $\mathrm{Mn}$ (whereas CO is not), and Mn concentration could conceivably fluctuate with SOD2 levels in brain tissue. However, transition metals are found in multiple cells types apart from neurons, and associating a single enzyme with a single metal may be futile. A more interesting and informative approach may be to compare metal distributions with inducible markers of neuronal activity such as c-FOS. ${ }^{39,40}$ The results of this study, although they do not provide detailed information about the function of the respective transition metals with respect to cortical regionalization, clearly show the feasibility of creating a cortical map based on metal concentration, which can be reliably aligned with maps based on other measured parameters. Ultimately, this may yield a new basis for understanding brain anatomy, in addition 
to classical histological methods. A key advantage of such an approach is the ability to monitor changes in metal distribution across the lifespan, which may provide relevant information for understanding normal and pathological brain functions.

Regional inhomogeneity of metal concentrations was not limited to the cortical compartment. In the hippocampus, there was dissociation between $\mathrm{Mn}$ and $\mathrm{Fe}$ in the local concentration maxima, with CA3 and the dentate gyrus exhibiting a pronounced increase in Mn concentration, while Fe was locally elevated in CA1 and nearby subiculum. It is premature to speculate on exactly why these fluctuations in metal concentration may occur, but it is worth noting that they were consistent across the animals studied, and there were regions outside the hippocampus where the relative concentrations of $\mathrm{Mn}$ and Fe were not in opposition (e.g. sensory cortex and putamen). Thus, the observed pattern does not reflect anticorrelation of $\mathrm{Mn}$ and Fe concentrations, but instead indicates that the functional mapping of metals will need to be considered on the basis of the functional, neurochemical, and anatomical characteristics of each area.

In general, the mean transition metal concentrations observed in the marmoset brain were consistent with the levels reported for other large laboratory species (Table 4), including sheep $^{41}$ and macaque (Mn only; ref. 42). However, the Mn and $\mathrm{Zn}$ levels observed in marmosets were somewhat lower than the values reported for human brain tissue, ${ }^{38,43}$ while the $\mathrm{Zn}$ concentrations were higher than those reported for discrete regions of the mouse brain. Finally, Fe concentrations in the macaque were higher than the equivalent areas of the sheep brain, although the overall range of values overlapped. The marmoset exhibited markedly higher Fe values than the mouse, ${ }^{44}$ though this may reflect technical or methodological differences between mouse studies (e.g. ref. 45). It may not be appropriate to directly compare tissue metal concentrations across species and methods, although the pattern of relative differences across brain compartments and subdivisions shows consistent patterns over multiple studies (Table 4). However, as investigators in anatomical disciplines become more aware of the possibilities for whole-brain metallomic mapping, these comparisons may become more feasible in the future. Here we show that the New World monkey is a promising model species for whole-brain metallomics, and holds great potential as a model species for analysis of experimentally induced and age-related neurodegeneration.

\section{Conflict of interest statement}

B. Paul maintains an ownership interest in Iolite Software, Inc. (makers of the BioLite package), and receives income from sale of this software. No other conflicts or potential conflicts are declared.

\section{Acknowledgements}

The authors thank Prof. Marcello Rosa, and Drs Elizabeth Zavitz, Maureen Hagen, Nicholas Price, and Leo Lui for sharing tissues from their electrophysiological experiments. Dr Zavitz and Ms Maree Reser provided helpful comments and proofreading of the manuscript prior to submission. Supported by: NHMRC Project Grant 1068140 (DHR); Ruhr University Bochum, Research School PLUS funded by Germany's Excellence Initiative [DFG GSC 98/3] (BK); ARC Linkage Project LP120200089 (DJH); Ramaciotti Foundation Establishment Grant (DJH); NHMRC Career Development Fellowship APP1122981 (DJH). The Florey Institute of Neuroscience and Mental Health acknowledges the strong support from the Victorian Government and in particular the funding from the Operational Infrastructure Support Grant.

\section{References}

1 A. A. Belaidi and A. I. Bush, Iron neurochemistry in Alzheimer's disease and Parkinson's disease: targets for therapeutics, J. Neurochem., 2016, 139(Suppl. 1), 179-197.

2 S. Ayton, P. Lei, D. J. Hare, J. A. Duce, J. L. George and P. A. Adlard, et al., Parkinson's disease iron deposition caused by nitric oxide-induced loss of beta-amyloid precursor protein, J. Neurosci., 2015, 35(8), 3591-3597.

3 S. L. Sensi, P. Paoletti, J. Y. Koh, E. Aizenman, A. I. Bush and M. Hershfinkel, The neurophysiology and pathology of brain zinc, J. Neurosci., 2011, 31(45), 16076-16085.

4 C. M. Opazo, M. A. Greenough and A. I. Bush, Copper: from neurotransmission to neuroproteostasis, Front. Aging Neurosci., 2014, 6, 143.

5 D. Hare, S. Ayton, A. Bush and P. Lei, A delicate balance: Iron metabolism and diseases of the brain, Front. Aging Neurosci., 2013, 5, 34.

6 S. A. James, D. J. Hare, N. L. Jenkins, M. D. de Jonge, A. I. Bush and G. McColl, phiXANES: In vivo imaging of metal-protein coordination environments, Sci. Rep., 2016, 6, 20350.

7 B. Paul, D. Hare, D. P. Bishop, C. Paton, V. T. Nguyen and N. Cole, et al., Visualising mouse neuroanatomy and function by metal distribution using laser ablation-inductively coupled plasma-mass spectrometry imaging, Chem. Sci., 2015, 6, 5383-5393.

8 A. B. Salmon, Moving toward "common" use of the marmoset as a non-human primate aging model, Pathobiol. Aging Age-Relat. Dis., 2016, 1, 4-6.

9 S. G. Solomon and M. G. P. Rosa, A simpler primate brain: the visual system of the marmoset monkey, Front. Neural Circuits, 2014, 8, 96.

10 E. Sasaki, H. Suemizu, A. Shimada, K. Hanazawa, R. Oiwa and M. Kamioka, et al., Generation of transgenic nonhuman primates with germline transmission, Nature, 2009, 459(7246), 523-527.

11 S. D. Tardif, K. G. Mansfield, R. Ratnam, C. N. Ross and T. E. Ziegler, The marmoset as a model of aging and agerelated diseases, ILAR J., 2011, 52(1), 54-65.

12 A. Lacreuse and J. G. Herndon, in Nonhuman Primate Models of Cognitive Aging, ed. J. L. Bizon and A. Woods, Humana Press, 2009, pp. 29-59.

13 R. Carrion and J. L. Patterson, An animal model that reflects human disease: The common marmoset (Callithrix jacchus), Curr. Opin. Virol., 2012, 2, 357-362. 
14 M.-F. Chesselet and S. T. Carmichael, Animal Models of Neurological Disorders, Neurotherapeutics, 2012, 9(2), 241-244.

15 G. Paxinos and C. Watson, George Paxinos Charles Watson., 1986.

16 K. B. Franklin and G. Paxinos, The Mouse Brain in Stereotaxic Coordinates, Academic Press (Elsevier), San Diego, CA, 2nd edn, 2004.

17 K. Zhang and T. J. Sejnowski, A universal scaling law between grey matter and white matter of cerebral cortex, Proc. Natl. Acad. Sci. U. S. A., 2000, 97(10), 5621-5626.

18 W. B. Hoover and R. P. Vertes, Anatomical analysis of afferent projections to the medial prefrontal cortex in the rat, Brain Struct. Funct., 2007, 212(2), 149-179.

19 B. A. Vogt and G. Paxinos, Cytoarchitecture of mouse and rat cingulate cortex with human homologies, Brain Struct. Funct., 2014, 219(1), 185-192.

20 S. Herculano-Houzel, C. E. Collins, P. Wong and J. H. Kaas, Cellular Scaling Rules for Primate Brains, Proc. Natl. Acad. Sci. U. S. A., 2007, 104(9), 3562-3567.

21 J. H. Kaas, The evolution of neocortex in primates, Prog. Brain Res., 2012, 195, 91-102.

22 K. J. Burman, D. H. Reser, H. H. Yu and M. G. Rosa, Cortical input to the frontal pole of the marmoset monkey, Cereb. Cortex, 2011, 21(8), 1712-1737.

23 D. H. Reser, K. J. Burman, K. E. Richardson, M. W. Spitzer and M. G. P. Rosa, Connections of the marmoset rostrotemporal auditory area: express pathways for analysis of affective content in hearing, Eur. J. Neurosci., 2009, 30(4), 578-592.

24 H. H. Yu, T. A. Chaplin, G. W. Egan, D. H. Reser, K. H. Worthy and M. G. Rosa, Visually evoked responses in extrastriate area MT after lesions of striate cortex in early life, J. Neurosci., 2013, 33(30), 12479-12489.

25 D. H. Reser, P. Majka, S. Snell, J. Chan, K. Watkins and K. Worthy, et al., Topography of claustrum and insula projections to medial prefrontal and anterior cingulate cortex of the common marmoset (Callithrix jacchus), J. Comp. Neurol., 2017, 525(6), 1421-1441.

26 F. Gallyas, Silver staining of myelin by means of physical development, Neurol. Res., 1979, 1(2), 203-209.

27 M. Wong-Riley, Changes in the visual system of monocularly sutured or enucleated cats demonstrable with cytochrome oxidase histochemistry, Brain Res., 1979, 171(1), 11-28.

28 R. B. H. Tootell and M. S. Silverman, Two methods for flatmounting cortical tissue, J. Neurosci. Methods, 1985, 15(3), 177-190.

29 M. G. Rosa, R. Gattass, M. Fiorani Jr. and J. G. Soares, Laminar, columnar and topographic aspects of ocular dominance in the primary visual cortex of Cebus monkeys, Exp. Brain Res., 1992, 88(2), 249-264.

30 G. Paxinos, C. Watson, M. Petrides, M. Rosa and H. Tokuno, The Marmoset Brain in Stereotaxic Coordinates, Academic Press, Elsevier, London, 2012.

31 S. Yuasa, K. Nakamura and S. Kohsaka, Stereotaxic atlas of the marmoset brain, 2010.

32 D. J. Hare, J. Lear, D. Bishop, A. Beavis and P. A. Doble, Protocol for production of matrix-matched brain tissue standards for imaging by laser ablation-inductively coupled plasma-mass spectrometry, Anal. Methods, 2013, 5(8), 1915-1921.

33 J. Lear, D. Hare, P. Adlard, D. Finkelstein and P. Doble, Improving acquisition times of elemental bio-imaging for quadrupole-based LA-ICP-MS, J. Anal. At. Spectrom., 2012, 27(1), 159-164.

34 D. Hare, C. Austin and P. Doble, Quantification strategies for elemental imaging of biological samples using laser ablation-inductively coupled plasma-mass spectrometry, Analyst, 2012, 137(7), 1527-1537.

35 C. Paton, J. Hellstrom, B. Paul, J. Woodhead and J. Hergt, Iolite: Freeware for the visualisation and processing of mass spectrometric data, J. Anal. At. Spectrom., 2011, 26(12), 2508-2518.

36 D. Colquhoun, An investigation of the false discovery rate and the misinterpretation of $p$ values, R. Soc. Open Sci., 2014, 1-15.

37 R. B. Tootell, S. L. Hamilton and M. S. Silverman, Topography of cytochrome oxidase activity in owl monkey cortex, J. Neurosci., 1985, 5(10), 2786-2800.

38 E. Bonilla, E. Salazar, J. J. Villasmil and R. Villalobos, The Regional Distribution of Manganese in the Normal Human, Neurochem. Res., 1982, 7(2), 221-227.

39 F. C. Cruz, E. Koya, D. H. Guez-Barber, J. M. Bossert, C. R. Lupica and Y. Shaham, et al., New technologies for examining the role of neuronal ensembles in drug addiction and fear, Nat. Rev. Neurosci., 2013, 14(11), 743-754.

40 E. Bullitt, Expression of c-fos-like protein as a marker for neuronal activity following noxious stimulation in the rat, J. Comp. Neurol., 1990, 296(4), 517-530.

41 S. Bakirdere, N. Kizilkan and M. Yaman, Determination of zinc, copper, iron, and manganese in different regions of lamb brain, Biol. Trace Elem. Res., 2011, 142(3), 492-499.

42 D. C. Dorman, M. F. Struve, M. W. Marshall, C. U. Parkinson, R. A. James and B. A. Wong, Tissue Manganese Concentrations in Young Male Rhesus Monkeys following Subchronic Manganese Sulfate Inhalation, Toxicol. Sci., 2006, 92(1), 201-210.

43 P. Ramos, A. Santos, N. R. Pinto, R. Mendes, T. Magalhães and A. Almeida, Anatomical Region Differences and Age-Related Changes in Copper, Zinc, and Manganese Levels in the Human Brain, Biol. Trace Elem. Res., 2014, 161(2), 190-201.

44 S. D. Portbury, D. J. Hare, C. Sgambelloni, D. I. Finkelstein and P. A. Adlard, A time-course analysis of changes in cerebral metal levels following a controlled cortical impact, Metallomics, 2016, 8(8), 193-200.

45 R. Rajendran, R. Minqin, M. D. Ynsa, G. Casadesus, M. A. Smith and G. Perry, et al., A novel approach to the identification and quantitative elemental analysis of amyloid deposits-Insights into the pathology of Alzheimer's disease, Biochem. Biophys. Res. Commun., 2009, 382(1), 91-95.

46 M. G. Rosa, S. M. Palmer, M. Gamberini, R. Tweedale, M. C. Pinon and J. A. Bourne, Resolving the organization of the New World monkey third visual complex: the dorsal extrastriate cortex of the marmoset (Callithrix jacchus), J. Comp. Neurol., 2005, 483(2), 164-191. 